\title{
Validation of repeated self-reported $n-3$ PUFA intake using serum phospholipid fatty acids as a biomarker in breast cancer patients during treatment
}

Sonja H. Brunvoll ${ }^{1,2^{*}}$ D, Inger Thune ${ }^{2,3}$, Hanne Frydenberg ${ }^{2}$, Vidar G. Flote ${ }^{2}$, Gro F. Bertheussen ${ }^{4,5}$, Ellen Schlichting ${ }^{6}$, Kristian S. Bjerve ${ }^{7,8}$ and Anette Hjartåker ${ }^{1}$

\begin{abstract}
Background: The role of n-3 polyunsaturated fatty acids (PUFAs) in breast cancer is not clear and under debate. To explore this relationship it is important to have proper validated dietary assessment methods for measuring the intake of n-3 PUFAs. The aim of the current study is to validate two different methods used to assess the intake of selected n-3 PUFAs as well as food sources of long-chained n-3 PUFAs. Also, we aim to study how stable the intake of fatty acids is during breast cancer treatment.
\end{abstract}

Methods: The study-population was patients with breast cancer (Stages I-II) or ductal carcinoma in situ (DCIS-grade III) undergoing treatment $(n=49)$ in Norway. Dietary intake was assessed by two self-administered methods, a 256 food item food frequency questionnaire (FFQ) and a 7-day pre-coded food diary (PFD). The FFQ was administered presurgery and twelve months postsurgery, and the PFD was administered shortly after surgery $(10+/-2$ days), six and twelve months postsurgery. Fasting blood samples (presurgery, six and twelve months postsurgery) were analysed for serum phospholipid fatty acids, a biomarker for intake of n-3 PUFAs.

Results: Mean (SD) age was 54.2 (7.8) years at diagnosis, and the mean (SD) body mass index (BMI) was 24.8 (3.4) $\mathrm{kg} / \mathrm{m}^{2}$. Correlation coefficients between dietary intakes of n-3 PUFAs measured with the FFQ and the PFD ranged from 0.35 to 0.66 . The correlation coefficients between the PFD and the biomarker (serum phospholipid n-3 PUFAs) as well as between the FFQ and the biomarker demonstrated stronger correlations twelve months after surgery ( $\rho$ 0.40-0.56 and 0.36-0.53, respectively) compared to around surgery ( $\rho 0.08-0.20$ and $0.28-0.38$, respectively). The same pattern was observed for intake of fatty fish. The intake of n-3 PUFAs did not change during treatment assessed by the FFQ, PFD or biomarker.

Conclusion: These results indicate that the FFQ and the PFD can be used to assess dietary intake of fish and n-3 PUFAs in breast cancer patients during breast cancer treatment. Still, the PFD shortly after surgery should be used with caution. The diet of patients undergoing breast cancer treatment was quite stable, and the intake of $n-3$ PUFAs did not change.

Keywords: Serum phospholipid fatty acids, Biomarker, Food frequency questionnaire, Pre-coded food diary, N-3 PUFAs, Fish, Validation, Breast cancer

\footnotetext{
* Correspondence: s.h.brunvoll@medisin.uio.no

${ }^{1}$ Department of Nutrition, Institute of Basic Medical Sciences, University of

Oslo, PO Box 1046 Blindern, 0317 Oslo, Norway

${ }^{2}$ The Cancer Centre, Oslo University Hospital Ullevål, Oslo, Norway

Full list of author information is available at the end of the article
}

(c) The Author(s). 2018 Open Access This article is distributed under the terms of the Creative Commons Attribution 4.0 International License (http://creativecommons.org/licenses/by/4.0/), which permits unrestricted use, distribution, and

reproduction in any medium, provided you give appropriate credit to the original author(s) and the source, provide a link to the Creative Commons license, and indicate if changes were made. The Creative Commons Public Domain Dedication waiver (http://creativecommons.org/publicdomain/zero/1.0/) applies to the data made available in this article, unless otherwise stated. 


\section{Background}

Knowledge of the role of individual fatty acids in health and disease is increasing, also when it comes to different types of cancers [1,2]. The role of $n-3$ polyunsaturated fatty acids (PUFAs) in the diet of women diagnosed with breast cancer is not clear [3-5]. However, it has been proposed that a high intake of $n-3$ PUFAs prediagnosis, as well as postdiagnosis, may improve prognosis and survival after breast cancer [2, 6-9]. Being diagnosed with breast cancer may lead to changes in the diet, due to a desire to cure the disease, alleviate symptoms of nausea or to follow healthcare professionals' advice [10].

To be able to investigate the n-3 PUFAs role in breast cancer prognosis or study dietary changes after diagnosis it is important to have proper methods to measure the dietary intake. Traditional methods for dietary assessment are usually based on self-report and include among other the food diary, which is usually conducted on several consecutive days. A food diary can provide detailed information about food intake, cooking methods, meals and eating frequency and can give relatively accurate information about dietary intake [11]. The weaknesses are that the tool is time-consuming both for the respondent and the investigator, multiple days are required to estimate habitual intake and the task of recording the diet may influence the dietary intake $[11,12]$. The food frequency questionnaire (FFQ) on the other hand attempts to estimate usual dietary intake in one administration, is usually a lower burden on the respondent and the investigator and the cost of administration and handling is relatively low [13]. However, the FFQ is a closed method and may give fewer details on specific food types and cooking methods, and is more prone to measurement errors including recall errors as it is memory-dependent [13].

Biomarkers may be used as objective indicators on dietary intake and can be used to validate dietary assessment methods [14]. Using serum phospholipid fatty acids as biomarkers have been shown to be useful to reflect dietary intake of fatty acids [15]. However, no fatty acid biomarker can reflect the exact fat intake as it is subject to absorption and endogenous metabolism and can be affected by factors such as hormones [14-20]. Certain phospholipid fatty acids such as the long-chained n-3 PUFAs provide a better reflection of the dietary intake, while the saturated (SFAs) and monounsaturated fatty acids (MUFAs) provide a weaker estimate of the dietary content because of a high degree of endogenous metabolism [16, 17]. The serum phospholipid fatty acids are short- to medium-term biomarkers that reflect the dietary intake of the individual fatty acids for the last days or weeks $[14,16,21]$.

It is important to have dietary assessment methods that are validated in the patient-group they are intended to be used. To our knowledge, methods assessing the intake of fatty acids in breast cancer patients during adjuvant breast cancer treatment have not previously been validated using a prospective method, a retrospective method and a biomarker. The aim of this study is to validate an FFQ and a pre-coded food diary (PFD) using serum phospholipid n-3 PUFAs as a biomarker for the intake of selected n-3 PUFAs as well as food-sources of long-chained n-3 PUFAs in breast cancer patients. By repeating our measurements over a year, we also examine how stable the intake of selected nutrients (including n-3 PUFAs) is during adjuvant breast cancer treatment.

\section{Methods}

\section{Subjects and study design}

A total of 60 women newly diagnosed with invasive histologically verified breast cancer (stages I-II) or ductal carcinoma in situ (DCIS-grade III) aged 35-75 years participated in a small clinical study between 2011 and 2013 at the Cancer Center, Oslo University Hospital Ullevål, Oslo; St.Olav University Hospital, Trondheim and Vestre Viken, Drammen, Norway. The patients were included before they underwent breast cancer surgery and were thereafter followed at the outpatient clinic. Patients with known severe illnesses (e.g. diabetes, heart disease) were excluded. A total of ten patients were excluded from the study after inclusion due to e.g. other diseases, unexpected settings in the family (e.g. death) or unable to participate due to their work setting $(n=50)$. One patient had incomplete dietary recordings, so finally 49 (82\%) patients completed all the dietary assessment and had available blood samples at all points up to 12 months after surgery and were included in the present analyses. A total of 45 patients had breast cancer and 4 patients had DCIS-grade III.

\section{Patient characteristics and clinical measurements}

The characteristics of the patients and clinical variables were assessed before any treatment (surgery, radiation, chemotherapy and/or endocrine therapy), including information regarding lifestyle habits, medical history and socioeconomic status. Anthropometric measurements were done with patients having no shoes on and wearing light clothes. Weight was measured to the nearest $0.1 \mathrm{~kg}$ on an electronic scale, height was measured to the nearest $0.5 \mathrm{~cm}$ and body mass index (BMI, $\mathrm{kg} / \mathrm{m}^{2}$ ) was calculated. Waist circumference was measured $2.5 \mathrm{~cm}$ above the umbilicus; hip circumference was measured at the widest point of the buttocks. Waist-to-hip ratio was calculated by dividing waist circumference by hip circumference. 


\section{Blood sample analyses}

Fasting (overnight) venous blood samples were drawn presurgery (2-8 days before surgery), six and 12 months postsurgery (Fig. 1). The blood was drawn into serum clot activator tubes with separation gel and left to clot at room temperature for $30 \mathrm{~min}$ before centrifugation at $2000 \mathrm{~g} / \mathrm{rcf}$ for $15 \mathrm{~min}$. After centrifugation the serum was aliquoted into $1 \mathrm{ml}$ cryotubes and left for $24 \mathrm{~h}$ at $20{ }^{\circ} \mathrm{C}$, before they were stored at $-80{ }^{\circ} \mathrm{C}$. In February 2014 the samples were transferred to the Department of Laboratory Medicine, St. Olav University Hospital, Trondheim, for analyses. Serum phospholipid fatty acid concentrations were measured as described previously [22]. Plasma proteins were precipitated using $70 \%(\mathrm{v} / \mathrm{v})$ perchloric acid (Merck) and total lipids extracted with n-butanol [23] using diheptadecanoyl-glycerophosphocholine and butylated hydroxytoluene (Sigma-Aldrich) as an internal standard and antioxidant, respectively. The phospholipids were isolated using Varian Bond Elute NH2 LRC columns and transmethylated in a $\mathrm{N}_{2}$ atmosphere using $\mathrm{BF}_{3}$ in methanol (Supelco Inc.) at a final concentration of $9.7 \%(w / w)$ at $135{ }^{\circ} \mathrm{C}$ for $30 \mathrm{~min}$. The fatty acid methyl esters were extracted into isooctane before analyzed by GLC on a Hewlett-Packard 5890A using a $30 \mathrm{~m}$ SP2330 fused silica capillary column, $0.25 \mathrm{~mm}$ internal diameter, $0.20 \mu \mathrm{m}$ film thickness (Supelco Inc.) with helium as a carrier at a linear gas velocity of $23 \mathrm{~cm} / \mathrm{sec}$. The initial column temperature was $170{ }^{\circ} \mathrm{C}$ programmed at $1.5{ }^{\circ} \mathrm{C} / \mathrm{min}$ to $210{ }^{\circ} \mathrm{C}$. The results were expressed as $\mathrm{mg}$ of phospholipid fatty acids per litre serum and recalculated to a percentage by weight (wt\%) on the basis of twenty-two identified fatty acids (Total fatty acids). A normal human serum sample was included in each run to monitor analytical performance. The between-series CVs for 18:0, $18: 1 n-9, \quad 18: 2 n-6, \quad 20: 3 n-6,20: 4 n-6, \quad 20: 5 n-3,22: 6 n-3$ and Total fatty acids were $3.3 \%, 4.9 \%, 3.1 \%, 4.0 \%$, $3.8 \%, 4.3 \%, 6.6 \%$ and $3.1 \%$ at a mean concentration of $169.5 \mathrm{mg} / \mathrm{L}, 117.7 \mathrm{mg} / \mathrm{L}, 214.5 \mathrm{mg} / \mathrm{L}, 18.1 \mathrm{mg} / \mathrm{L}$, $120.0 \mathrm{mg} / \mathrm{L}, 68.7 \mathrm{mg} / \mathrm{L}, 121.8 \mathrm{mg} / \mathrm{L}$ and $1246.0 \mathrm{mg} / \mathrm{L}$, respectively. The fatty acids examined in this study were the long-chained PUFAs EPA (eicosapentaenoic acid, 20:5n-3), DHA (docosahexaenoic acid, 22:6n-3) and the sum of the following n-3 PUFAs (hereafter termed "sum n-3"); ALA (alpha-linolenic acid, 18:3n-3), EPA, DPA (docosapentaenoic acid, 22:5n-3) and DHA.

\section{FFQ and PFD}

Two different dietary assessment methods were used; an FFQ and a PFD. A modified version of an FFQ developed and validated by the Department of Nutrition, Institute of Basic Medical Sciences, University of Oslo, was used to gather data on the usual diet (retrospective data) [24-27]. The FFQ is 14 pages and includes 256 questions about food items arranged after the traditional meal pattern in Norway. Serving size per consumption is given in various household units such as spoons, deciliter, cups, glasses and slices and frequency of food items consumed range from never/seldom to several times a day. The types of fat used in cooking and as spread on bread are covered in separate sections, as is questions regarding dietary supplements usage, such as fish oil capsules and cod liver oil. At the end of the FFQ, there are open spaces where the patients can fill in food items or dietary supplements not covered in the questionnaire. Presurgery, the patients were asked to report their usual diet during the last year before they were diagnosed with breast cancer or DCIS-grade III. Twelve months after surgery the patients were asked to report their diet for the last month (Fig. 1). The questionnaires were the same, although covering different time-periods.

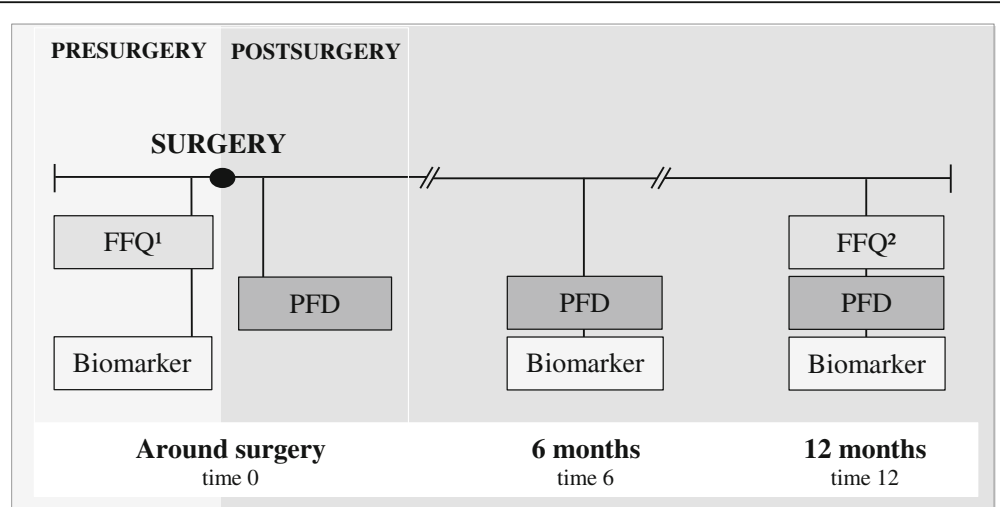

Fig. 1 Timeline for FFQ, PFD and biomarker (serum phospholipid fatty acids). 'FFQ asks for the last year's diet, filled in 2-8 days before surgery. ${ }^{2} \mathrm{FFQ}$ asks for the last month's diet. The PFD is filled in for seven consecutive days shortly after surgery $(10+/-2$ days after surgery), at six and twelve months postsurgery. The blood samples (biomarker) are drawn 2-8 days presurgery, at six and twelve months postsurgery 
The patients also recorded all their food and drink consumption in a modified version of a PFD developed and validated by the Department of Nutrition, Institute of Basic Medical Sciences, University of Oslo [28-30]. The PFD is 19 pages and includes 310 questions on consumption of different food items arranged and grouped after the regular Norwegian meal pattern. Each food group has an open space at the end where food items/ dishes and dietary supplements not covered in the pre-coded part can be listed. The design of the PFD is presented like a cross-table with time span at the top and type of food on the left side. Each day is divided into five time periods: 4 day periods where each is for $4 \mathrm{~h}$ (e.g. 6-10) and one night period from 22 to 06 . Household units and photos from a validated photo-booklet were used to estimate the amounts consumed [31]. The photo-booklet contains 15 colour-photo series of different types of food, each with four serving sizes spanning from small to a large portion. The patients recorded the food they had eaten by filling in the type of food and the number of items eaten in the corresponding time period. The PFD was filled in for seven consecutive days shortly after surgery $(10+/-2$ days after surgery), 6 months postsurgery and 12 months postsurgery (that is $3 \times 7$ days, Fig. 1).

Both dietary assessment methods (FFQ and PFD) were obtained by self-report. However, all FFQs and PFDs were then manually checked by trained personnel; in case of inconsistencies or missing information the patients were contacted and the missing values were obtained. The completed FFQs and PFDs were scanned using the Cardiff TeleForm program version 10.5.1 (Datascan Oslo, Norway). Food and nutrient calculations, as well as fatty acid intake, were computerised using the food database AE-14 and the KBS calculation software system (Kostberegningsystem) at the Department of Nutrition, Institute of Basic Medical Sciences, University of Oslo. The food database AE-14 is based on the Norwegian food composition tables from 2014 and 2015 (http://www.norwegianfoodcomp.no/), supplemented with data from calculated recipes and other databases, and has a large number of fatty acids including n-3 PUFAs. The n-3 PUFAs that were investigated from the diet were the long-chained PUFAs EPA and DHA in addition to the total n-3 PUFAs. The "sum n-3"-variable (used for the biomarker; the sum of ALA, EPA, DPA and DHA) was not used for the diet as it was not considered relevant. The "sum n-3" variable accounts for approximately $80-90 \%$ of total $n-3$ PUFAs in the diet and hence the variable "total n-3" was used for diet. The food categories "fatty fish" (all fatty fish in the diet, e.g. salmon, trout, mackerel and herring) and "fish and fish products" (all fatty and lean fish as well as fish products, sushi and shellfish) were also examined.

\section{Statistical analyses}

All analyses in this study are based on subjects who had completed all three assessments on all visits (PFD, FFQ and biomarker; $n=49$ ). The sample size required when expecting a correlation coefficient around 0.40, with $80 \%$ statistical power and 5\% significance level, is 46 subjects [32]. Statistical analyses were performed with IBM SPSS Statistics version 24.

Energy percent from macronutrients were calculated (E \%). Median dietary intakes in the PFD and the FFQ, as well as median serum phospholipid fatty acids around surgery, 6 months and 12 months, were calculated and compared using Wilcoxon signed-rank test as the data distribution was skewed. The agreement between the methods was determined by cross-classification into tertiles. Spearman correlation coefficients were calculated between PFD and FFQ, PFD and biomarker (serum phospholipid fatty acids) and FFQ and biomarker. The 95\% confidence intervals (CI) for the correlation coefficients were calculated using the Fisher $\mathrm{Z}$ method.

Spearman correlation coefficients were also calculated between the intake of EPA and DHA in the PFD/FFQ and the level of EPA and DHA in serum phospholipids, stratified by chemotherapy (yes/no), radiation (yes/no), endocrine therapy (yes/no), premenopausal/postmenopausal status, alcohol intake (median split) and BMI $(<25 / 25 \leq)$. The Fisher's $\mathrm{Z}$ transformation was used to investigate if there were any statistically significant differences in correlation coefficients between the strata. If any significant differences were detected between strata, Mann-Whitney test was performed to see if there were any differences in intake or in serum phospholipid fatty acids. A significance criterion of $p<0.05$ was used.

\section{Results}

Characteristics of the patients are presented in Table 1. The patients were on average 54.2 years at diagnosis (range 38-69), and their mean body mass index (BMI) was $24.8 \mathrm{~kg} / \mathrm{m}^{2}$ (range 20.2-33.2). The majority of the patients received radiotherapy (38 patients, 78\%), 30 patients (61\%) received chemotherapy and 31 (63\%) endocrine therapy.

The results from analysing serum phospholipid fatty acids are presented in Table 2 . None of the fatty acids, expressed as $w t \%$ of total fatty acids in serum phospholipids, changed significantly over time. The full fatty acid composition is presented in the Additional file 1: Table S1.

\section{FFQ compared to PFD}

The dietary intakes reported by the FFQ and PFD around surgery, at six and 12 months postsurgery are given in Table 3 . When comparing dietary intake recorded around surgery, the median intake in the FFQ compared to the PFD was significantly higher for most 
Table 1 Descriptive statistics of the patients by means, SD and range, $n=49$

\begin{tabular}{llll}
\hline Characteristics presurgery & Mean & SD & Range \\
\hline Age at diagnosis, years & 54.2 & 7.8 & $38-69$ \\
Education, years* & 16.2 & 3.4 & $8-24$ \\
Postmenopausal, no (\%) & $32(65)$ & & \\
Smoking, no (\%) & $9(18)$ & & \\
Height, cm & 168.1 & 5.9 & $155-181$ \\
Weight, kg & 70.4 & 11.7 & $48.5-97.1$ \\
BMl, kg/m & 24.8 & 3.4 & $20.2-33.2$ \\
Waist circumference, cm & 86.8 & 11.1 & $69.0-112.5$ \\
Waist/hip ratio & 0.86 & 0.06 & $0.75-1.00$ \\
Treatment (around 6-12 months) & & & \\
$\quad$ Radiotherapy, no (\%) & $38(78)$ & & \\
$\quad$ Chemotherapy, no (\%) & $30(61)$ & & \\
$\quad$ Endocrine therapy, no (\%) & $31(63)$ & & \\
SD Standard deviation & & & \\
* $_{n}=$ 48 & &
\end{tabular}

nutrients, including total PUFA, total $n-6$ and $n-3$ PUFAs, EPA and DHA. When looking at E \% from each nutrient most nutrients remained significantly higher in the FFQ, but total PUFA and total n-6 PUFA were no longer significantly different in the two methods. E \% from total and saturated fat intake was higher in the PFD. The dietary intake at 12 months demonstrated a similar pattern as seen around surgery, with generally significantly higher intake measured with the FFQ including total PUFA, total $n-6$ and n-3 PUFAs, EPA and DHA and n-3 PUFAs from supplements. Looking at $\mathrm{E} \%$ from each nutrient, most nutrients remained higher in the FFQ but there was no longer a significant difference in intake of total n-6 PUFAs and n-3 PUFAs from supplements. Also, E \% from total and saturated fat was significantly higher in the PFD.

\section{Dietary changes}

When studying each dietary assessment method separately, most nutrients did not change over time (Table 3). For the PFD the intake of total and saturated fat, as well as total n-6 PUFAs significantly decreased from shortly after surgery to 6 months postsurgery. The intake of sugar significantly decreased from shortly after surgery to 12 months postsurgery, and the intake of fish and fish products significantly decreased from 6 months to 12 months postsurgery. For the FFQ, the only significant change was a decreased intake of sugar and alcohol from the year before diagnosis to 12 months postsurgery.

\section{Fish intake}

In the PFD, 46 patients shortly after surgery, 49 at six and 48 at 12 months postsurgery had eaten fish or fish products during the 7 days of diet-registration. The median daily intake of fish and fish products were 73,79 and $67 \mathrm{~g}$, respectively, of which 21, 16 and 26 g were fatty fish (Table 3). Around surgery, the Spearman correlation coefficients (Table 4) between intake of fish and fish products recorded by the PFD and the biomarker as well as fatty fish intake and biomarker, ranged from 0.14 to 0.25 . At 6 months, the correlation coefficients between intake of fish and fish products and biomarker ranged from 0.24 to 0.33 and for fatty fish and biomarker from 0.15 to 0.27 . At 12 months, the correlation coefficients between intake of fish and fish products and biomarker ranged from 0.16 to 0.27 and for fatty fish and biomarker from 0.30 to 0.40 .

In the FFQ, the median daily intake of fish and fish products was $77 \mathrm{~g}$ the year before diagnosis and $79 \mathrm{~g}$ at 12 months, while intake of fatty fish was 21 and $20 \mathrm{~g}$, respectively. All but one patient reported consuming fish or fish products the year before diagnosis and all patients reported consuming fish or fish products at 12 months. The Spearman correlation coefficients (Table 4) between intake of fish and fish products and biomarker presurgery ranged from 0.03 to 0.05 , while fatty fish intake and biomarker ranged from 0.16 to 0.22 . At 12 months the correlation coefficients for the intake of fish and fish products and biomarker ranged from 0.13 to 0.23 and for fatty fish intake and biomarker from 0.31 to 0.44 .

\section{Supplements with $\mathrm{n}-3$ PUFAs}

About $70-85 \%$ of the supplements reported contained n-3 PUFAs and the supplements were a substantial contributor to total n-3 PUFAs, EPA and DHA intake in the current study. In the PFD, the number of patients taking

Table 2 The biomarker serum phospholipid fatty acids in wt $\%$ and quartiles $\left(P_{25}, 75\right), n=49$

\begin{tabular}{|c|c|c|c|c|c|c|}
\hline \multirow{2}{*}{$\begin{array}{l}\text { Fatty acid, } \\
\text { wt } \%\end{array}$} & \multicolumn{2}{|l|}{ Time 0} & \multicolumn{2}{|l|}{ Time 6} & \multicolumn{2}{|l|}{ Time 12} \\
\hline & Median & $P_{25,75}$ & Median & $P_{25,75}$ & Median & $P_{25,75}$ \\
\hline Sum n-3 & 10.24 & $8.03-11.54$ & 9.33 & $8.07-11.12$ & 9.31 & $7.99-10.85$ \\
\hline EPA & 2.54 & $1.84-3.17$ & 2.23 & $1.56-2.79$ & 2.19 & $1.53-3.03$ \\
\hline DHA & 6.24 & $5.44-7.13$ & 6.10 & $5.13-6.73$ & 5.84 & $4.97-6.72$ \\
\hline
\end{tabular}

wt\%: Weight percent

time $0: 2-8$ days presurgery, time $6: 6$ months postsurgery, time 12: 12 months postsurgery

${ }^{\dagger}$ Sum n-3: ALA (18:3n-3), EPA (20:5n-3), DPA (22:5n-3), DHA (22:6n-3) 


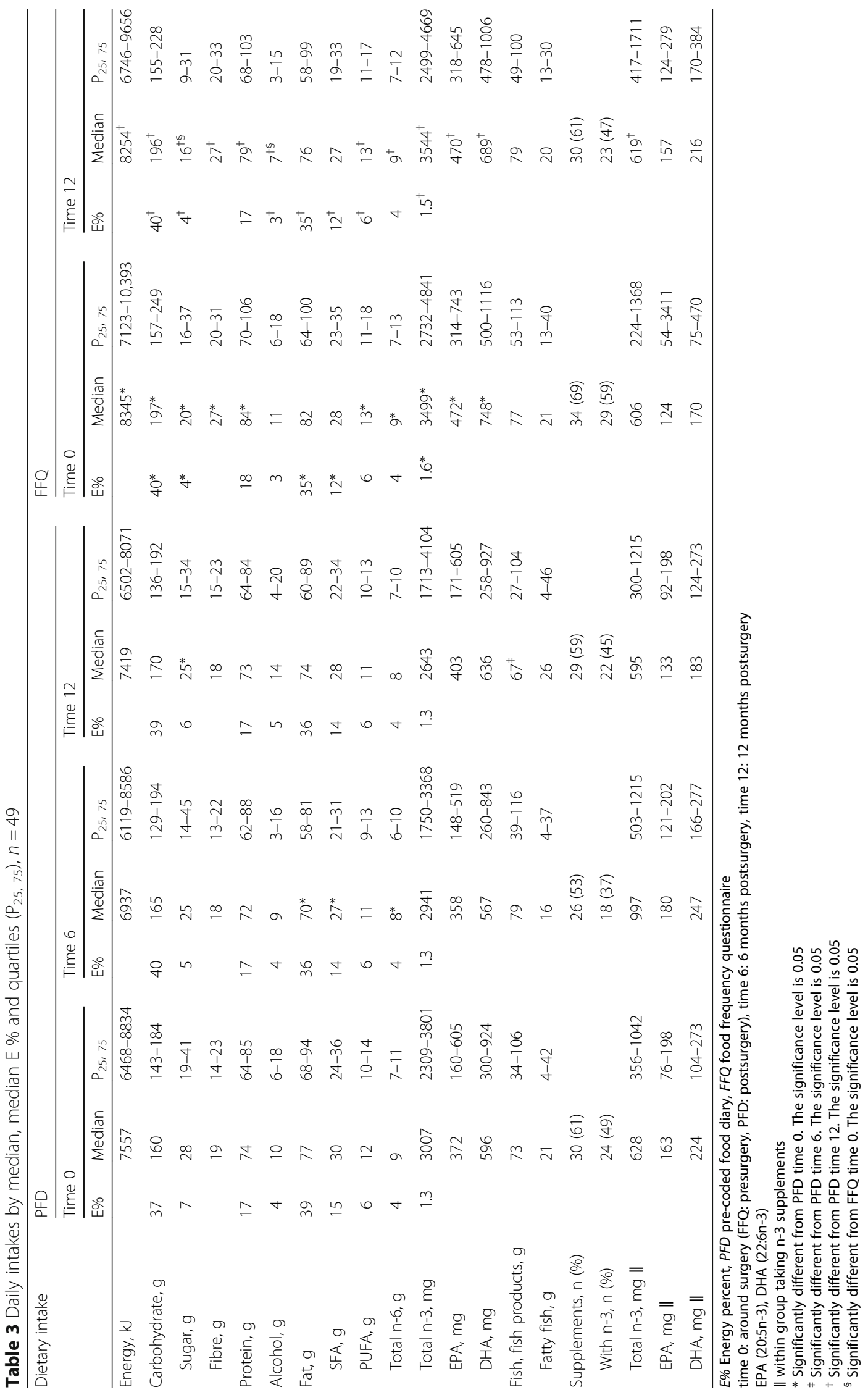


Table 4 Spearman correlation coefficients ( $\rho$ ) between fish/fish products and fatty fish and biomarker, $n=49$

\begin{tabular}{|c|c|c|c|c|c|c|c|c|c|}
\hline \multirow[t]{3}{*}{ Biomarker } & \multirow[b]{3}{*}{ Timeł } & \multicolumn{4}{|l|}{ PFD } & \multicolumn{4}{|l|}{ FFQ } \\
\hline & & \multicolumn{2}{|c|}{ Fish and fish products } & \multicolumn{2}{|c|}{ Fatty fish } & \multicolumn{2}{|c|}{ Fish and fish products } & \multicolumn{2}{|c|}{ Fatty fish } \\
\hline & & $\rho$ & $95 \% \mathrm{Cl}$ & $\rho$ & $95 \% \mathrm{Cl}$ & $\rho$ & $95 \% \mathrm{Cl}$ & $\rho$ & $95 \% \mathrm{Cl}$ \\
\hline \multirow[t]{3}{*}{ Sum n-3† } & 0 & 0.19 & $-0.10,0.45$ & 0.21 & $-0.07,0.47$ & 0.03 & $-0.25,0.31$ & 0.16 & $-0.13,0.42$ \\
\hline & 6 & $0.32^{*}$ & $0.04,0.55$ & 0.27 & $-0.01,0.52$ & & & & \\
\hline & 12 & 0.24 & $-0.04,0.49$ & $0.40^{*}$ & $0.14,0.61$ & 0.20 & $-0.09,0.46$ & $0.43^{*}$ & $0.17,0.64$ \\
\hline \multirow[t]{3}{*}{ EPA } & 0 & 0.14 & $-0.15,0.40$ & 0.18 & $-0.11,0.44$ & 0.05 & $-0.23,0.33$ & 0.20 & $-0.09,0.45$ \\
\hline & 6 & 0.24 & $-0.04,0.49$ & 0.15 & $-0.14,0.41$ & & & & \\
\hline & 12 & 0.16 & $-0.13,0.42$ & $0.39^{*}$ & $0.12,0.60$ & 0.13 & $-0.16,0.39$ & $0.31^{*}$ & $0.03,0.54$ \\
\hline \multirow[t]{3}{*}{ DHA } & 0 & 0.25 & $-0.03,0.50$ & 0.23 & $-0.05,0.48$ & 0.04 & $-0.25,0.32$ & 0.22 & $-0.07,0.47$ \\
\hline & 6 & $0.33^{*}$ & $0.05,0.56$ & 0.26 & $-0.03,0.50$ & & & & \\
\hline & 12 & 0.27 & $-0.02,0.51$ & $0.30^{*}$ & $0.02,0.54$ & 0.23 & $-0.06,0.48$ & $0.44^{*}$ & $0.19,0.65$ \\
\hline
\end{tabular}

Cl Confidence interval, PFD pre-coded food diary, FFQ food frequency questionnaire

†Sum n-3: ALA (18:3n-3), EPA (20:5n-3), DPA (22:5n-3), DHA (22:6n-3)

ftime 0: around surgery (FFQ: presurgery, PFD: postsurgery, biomarker: presurgery), time 6: 6 months postsurgery, time 12 : 12 months postsurgery

${ }^{*} p<0.05$

supplements with n-3 PUFAs during diet-registration was 24,18 and 22 shortly after surgery, at six- and 12 months postsurgery, respectively. The correlation coefficients between intake of total n-3 PUFAs, EPA and DHA from supplements and corresponding fatty acids in serum phospholipids around surgery ranged from 0.03 to 0.06 . At six and 12 months the range was from 0.33 to 0.58 (Additional file 1: Table S2). In the FFQ, 29 patients reported taking supplements containing $n-3$ PUFAs the year before diagnosis and 23 reported taking it at 12 months. The correlation coefficients between total n-3 PUFAs, EPA and DHA from supplements and the corresponding fatty acids in serum phospholipids ranged from 0.34 to 0.54 at both time-points (See Additional file 1: Table S2).

\section{N-3 PUFAs}

The Spearman correlation coefficients between dietary intake of n-3 PUFAs, EPA and DHA and the fatty acids in serum phospholipids are shown in Table 5. The correlations between the FFQ and the PFD ranged from 0.46 to 0.53 for total n-3 PUFAs, EPA and DHA around surgery and 12 months postsurgery. The Spearman correlation coefficients between the PFD and the biomarker around surgery ranged from 0.10 to 0.19 , at 6 months the correlation coefficients ranged from 0.32 to

Table 5 Spearman correlation coefficients $(\rho)$ and cross-classification for selected fatty acids, $n=49$

\begin{tabular}{|c|c|c|c|c|c|c|c|c|c|c|c|c|c|}
\hline \multirow[t]{3}{*}{ Fatty acid } & \multirow[b]{3}{*}{ timeł } & \multicolumn{4}{|c|}{ FFQ v. PFD } & \multicolumn{4}{|c|}{ PFD v. Biomarker } & \multicolumn{4}{|c|}{ FFQ v. Biomarker } \\
\hline & & \multirow[b]{2}{*}{$\rho$} & \multirow[b]{2}{*}{$95 \% \mathrm{Cl}$} & \multicolumn{2}{|c|}{ Cross-classification } & \multirow[b]{2}{*}{$\rho$} & \multirow[b]{2}{*}{$95 \% \mathrm{Cl}$} & \multicolumn{2}{|c|}{ Cross-classification } & \multirow[b]{2}{*}{$\rho$} & \multirow[b]{2}{*}{$95 \% \mathrm{Cl}$} & \multicolumn{2}{|c|}{ Cross-classification } \\
\hline & & & & ST & OT & & & ST & OT & & & ST & OT \\
\hline \multirow[t]{3}{*}{$n-3+$} & 0 & $0.50^{*}$ & $0.26,0.69$ & 53 & 10 & 0.19 & $-0.09,0.45$ & 35 & 16 & $0.28^{*}$ & $0.00,0.52$ & 37 & 14 \\
\hline & 6 & & & & & $0.40^{*}$ & $0.13,0.61$ & 45 & 14 & & & & \\
\hline & 12 & $0.51^{*}$ & $0.27,0.69$ & 47 & 8 & $0.56^{*}$ & $0.33,0.73$ & 57 & 6 & $0.50^{*}$ & $0.26,0.69$ & 47 & 8 \\
\hline \multirow[t]{3}{*}{ EPA } & 0 & $0.53^{*}$ & $0.30,0.71$ & 57 & 6 & 0.10 & $-0.18,0.37$ & 35 & 16 & $0.30^{*}$ & $0.02,0.54$ & 45 & 14 \\
\hline & 6 & & & & & $0.32^{*}$ & $0.04,0.55$ & 33 & 10 & & & & \\
\hline & 12 & $0.46^{*}$ & $0.20,0.65$ & 55 & 12 & $0.56^{*}$ & $0.33,0.73$ & 53 & 6 & $0.38^{*}$ & $0.11,0.60$ & 41 & 10 \\
\hline \multirow[t]{3}{*}{ DHA } & 0 & $0.51^{*}$ & $0.27,0.69$ & 59 & 8 & 0.17 & $-0.12,0.43$ & 33 & 14 & $0.33^{*}$ & $0.05,0.56$ & 41 & 14 \\
\hline & 6 & & & & & $0.38^{*}$ & $0.11,0.60$ & 59 & 12 & & & & \\
\hline & 12 & $0.48^{*}$ & $0.23,0.67$ & 47 & 12 & $0.44^{*}$ & $0.18,0.64$ & 51 & 8 & $0.51^{*}$ & $0.27,0.69$ & 55 & 8 \\
\hline
\end{tabular}

CI Confidence interval, PFD pre-coded food diary, FFQ food frequency questionnaire Cross-classification, \% classified in ST: same tertile, OT: opposite tertile, grossly misclassified tn-3: sum n-3 (ALA (18:3n-3), EPA (20:5n-3), DPA (22:5n-3), DHA (22:6n-3)) for biomarker and total n-3 PUFAs for PFD/FFQ \#time 0: around surgery (FFQ: presurgery, PFD; postsurgery, biomarker; presurgery), time 6: 6 months postsurgery, time 12: 12 months postsurgery * $p<0.05$ 
0.40 and at 12 months from 0.44 to 0.56 . For the FFQ, Spearman correlation coefficients with the biomarker presurgery ranged from 0.28 to 0.33 and at 12 months it ranged from 0.38 to 0.51 .

When studying $\mathrm{E} \%$ from the n-3 PUFAs (Table 6) most of the correlation coefficients were not altered much, except for a stronger correlation coefficient for total n-3 PUFAs between FFQ and PFD around surgery (from 0.50 to 0.66 ) and a weaker correlation for EPA and DHA at 12 months (from 0.46 to 0.35 and from 0.48 to 0.38 , respectively). The correlation coefficient for DHA at 12 months between FFQ (E \%) and biomarker was also weaker than for crude intake (0.41 v. 0.51).

Cross-classification of patients by tertiles of reported intakes and serum phospholipid n-3 PUFA, EPA and DHA are shown in Tables 5 and 6. The percentages of patients categorized in the same tertile were generally higher at 12 months than around surgery, for PFD/biomarker $45-57 \%$ v. $31-41 \%$ and for FFQ/biomarker $41-$ $57 \%$ v. $37-49 \%$, respectively. Furthermore, the percentages of patients categorized in the opposite tertile, that is grossly misclassified, were generally lower at 12 months than around surgery, for PFD/biomarker 6-10\% v. $14-16 \%$, respectively. For FFQ/biomarker this pattern was not apparent with 10-14\% grossly misclassified presurgery and $8-14 \%$ at 12 months postsurgery.

We also examined correlation coefficients according to patient characteristics (e.g. menopausal status or BMI), and treatment regime. The only significant difference was when stratified by chemotherapy (yes/no) for intake of EPA measured with the PFD and EPA in serum phospholipid at 6 months $(p=0.002)$. The Spearman correlation coefficient for the patients undergoing chemotherapy $(n=30)$ was $\rho-0.05$ and for the patients not undergoing chemotherapy $(n=19) \rho 0.73$. MannWhitney test comparing intake of EPA and EPA in serum phospholipids between the two strata demonstrated a significantly lower level of EPA in serum phospholipids in the patients undergoing chemotherapy $(2.0$ v. $2.6 \mathrm{wt} \%, p=0.03)$ but no difference in intake of EPA.

\section{Discussion}

In this study, we observed acceptable/moderate correlations between dietary intakes of total n-3 PUFAs, EPA and DHA measured with the FFQ and PFD. The correlations between the PFD and biomarker as well as between the FFQ and biomarker were stronger at 12 months postsurgery than around surgery. The same pattern was observed for fatty fish. Intake of most nutrients, including n-3 PUFAs, did not change during treatment assessed by the FFQ or PFD.

The median intakes of macronutrients were in general significantly higher in the FFQ than in the PFD, both around surgery and 12 months postsurgery. In comparison, other studies have demonstrated conflicting results when comparing an FFQ to a reference method. However, most often the FFQ gives higher estimates of macronutrient intake as well as intake of PUFAs $[20,26,33-38]$. A study among elderly (age 6780 years) Norwegian women demonstrated a similar pattern as the present study with generally higher macronutrient intakes reported by a similar FFQ compared to weighed food records [26].

The intake of fish and fish products, as well as fatty fish, did not differ between the two dietary methods in the present study. Compared to the intake of fish and fish products reported by Norwegian women participating in the national Norkost 3 survey (age 18-70 years)

Table 6 Spearman correlation coefficients $(\rho)$ and cross-classification for selected fatty acids, energy percent $(E \%) n=49$

\begin{tabular}{|c|c|c|c|c|c|c|c|c|c|c|c|c|c|}
\hline \multirow[t]{3}{*}{ Fatty acid } & \multirow[b]{3}{*}{ timeł } & \multicolumn{4}{|c|}{ FFQ v. PFD } & \multicolumn{4}{|c|}{ PFD v. Biomarker } & \multicolumn{4}{|c|}{ FFQ v. Biomarker } \\
\hline & & \multirow[b]{2}{*}{$\rho$} & \multirow[b]{2}{*}{$95 \% \mathrm{Cl}$} & \multicolumn{2}{|c|}{ Cross-classification } & \multirow[b]{2}{*}{$\rho$} & \multirow[b]{2}{*}{$95 \% \mathrm{Cl}$} & \multicolumn{2}{|c|}{ Cross-classification } & \multirow[b]{2}{*}{$\rho$} & \multirow[b]{2}{*}{$95 \% \mathrm{Cl}$} & \multicolumn{2}{|c|}{ Cross-classification } \\
\hline & & & & ST & OT & & & ST & OT & & & ST & OT \\
\hline \multirow[t]{3}{*}{$n-3+$} & 0 & $0.66^{*}$ & $0.47,0.80$ & 55 & 4 & 0.20 & $-0.08,0.46$ & 41 & 14 & $0.33^{*}$ & $0.06,0.56$ & 39 & 12 \\
\hline & 6 & & & & & $0.39^{*}$ & $0.12,0.60$ & 45 & 12 & & & & \\
\hline & 12 & $0.48^{*}$ & $0.23,0.67$ & 57 & 10 & $0.52^{*}$ & $0.28,0.70$ & 49 & 6 & $0.53^{*}$ & $0.29,0.70$ & 57 & 10 \\
\hline \multirow[t]{3}{*}{ EPA } & 0 & $0.58^{*}$ & $0.35,0.74$ & 51 & 4 & 0.08 & $-0.21,0.35$ & 31 & 16 & $0.35^{*}$ & $0.08,0.58$ & 49 & 10 \\
\hline & 6 & & & & & 0.27 & $-0.01,0.51$ & 37 & 16 & & & & \\
\hline & 12 & $0.35^{*}$ & $0.08,0.57$ & 53 & 18 & $0.51^{*}$ & $0.26,0.69$ & 55 & 8 & $0.36^{*}$ & $0.09,0.58$ & 49 & 14 \\
\hline \multirow[t]{3}{*}{$\mathrm{DHA}$} & 0 & $0.59^{*}$ & $0.36,0.74$ & 59 & 8 & 0.18 & $-0.11,0.44$ & 41 & 14 & $0.38^{*}$ & $0.11,0.60$ & 43 & 12 \\
\hline & 6 & & & & & $0.39^{*}$ & $0.12,0.60$ & 53 & 14 & & & & \\
\hline & 12 & $0.38^{*}$ & $0.11,0.60$ & 47 & 16 & $0.40^{*}$ & $0.13,0.61$ & 45 & 10 & $0.41^{*}$ & $0.14,0.62$ & 43 & 8 \\
\hline
\end{tabular}

Cl Confidence interval, PFD pre-coded food diary, FFQ food frequency questionnaire Cross-classification, \% classified in ST: same tertile, OT: opposite tertile, grossly misclassified

tn-3: sum n-3 (ALA (18:3n-3), EPA (20:5n-3), DPA (22:5n-3), DHA (22:6n-3)) for biomarker and total n-3 PUFAs for PFD/FFQ

ftime 0: around surgery (FFQ: presurgery, PFD; postsurgery, biomarker; presurgery), time 6: 6 months postsurgery, time 12 : 12 months postsurgery

* $p<0.05$ 
the patients in our study reported around 20-40\% higher fish intake (depending on time-point). However, fish intake in Norkost 3 is based on two 24 h recalls which might be too few administrations to give a good estimate on average fish-intake [39].

The intake of n-3 PUFAs among the patients in our study was higher than reported by others $[6,40]$. The intake of total n-3 PUFAs was more than three times higher, and the intake of EPA and DHA was more than ten times higher than in an American study among newly diagnosed breast cancer patients [6]. This latter study is consistent with an other American study among women at risk for breast cancer [40]. Further, EPA and DHA in serum phospholipids were higher in patients in the present study compared to breast cancer patients in a Swedish study measured prediagnostic [41].

The correlations between intake of total n-3 PUFAs, EPA and DHA measured with the FFQ and PFD were acceptable/moderate and higher around surgery than at 12 months postsurgery. The correlations were within the same range or higher than demonstrated in validation studies comparing FFQ to dietary records, weighed food records or $24 \mathrm{~h}$ recalls in healthy adults [20, 42-44].

When comparing the PFD intake of total n-3 PUFAs, EPA and DHA with corresponding fatty acids in serum phospholipids, the timing of measurement was of great importance. It is clear that the correlation coefficients between PFD and serum phospholipid fatty acids were low around surgery, higher at 6 months and the highest correlation was observed at 12 months postsurgery. There might be several reasons for this pattern. At six and 12 months, the PFD was usually distributed the same day or one of the nearby days as the blood sampling, meaning that the biomarker represented the diet days/weeks before it was measured with the PFD. Around surgery, the blood sample was drawn 2-8 days before surgery, and the PFD was distributed $10+/-$ 2 days postsurgery. Due to different timing, especially around surgery, the correlation coefficients observed might be underestimates of the true correlations. It is uncertain what kind of impact the breast cancer diagnosis or breast cancer surgery might have had on the actual diet around surgery or on filling in the quite extensive PFD. What is certain is that a breast cancer diagnosis can induce stress, and the level of stress might change from the time of diagnosis throughout the treatment period $[45,46]$. It might be that our patients actually ate differently or changed their diet at the time of filling in the PFD compared to when the blood samples were drawn, although there were minimal changes throughout the year postsurgery. When it comes to filling in the PFD, it can be questioned if the workload of that task was too much, or that the patients could not put focus into it so soon after diagnosis. Also, repeated administrations of the PFD would pose a learning-effect in both the respondent and the study personnel throughout the study period.

With the FFQ, the timing of blood sampling and mapping of diet coincided better than for the PFD because of the retrospective nature of the FFQ. Both presurgery and 12 months postsurgery, the FFQ was distributed at the same time as the blood sample was drawn. The difference between the two time-points is that FFQ presurgery represented last year's diet and at 12 months it represented the last month while the biomarker at both points represented the intake the last days or weeks. This might be an explanation for the stronger correlations seen at 12 months. Previously, studies have demonstrated that length of the reference period for the FFQ is of importance; the FFQs that report the diet the last month have somewhat higher correlations with the reference method in comparison to the ones reporting the diet the last year [47].

Even though the setting of our study was patients with breast cancer or DCIS-grade III undergoing treatment, the diet seemed quite stable. In particular, the dietary intake of total n-3 PUFAs, EPA and DHA in the FFQ and PFD did not change over the year. In the FFQ, only the intake of sugar and alcohol decreased from prediagnosis to 12 months postsurgery. The decrease in alcohol intake might have been due to the advice on restricting alcohol intake to a moderate level. On the contrary, such a decrease was not seen in the PFD and absolute intake of alcohol was also higher in the PFD at 12 months compared to FFQ, but no difference around surgery. We may question whether this difference in reporting of alcohol intake is a social desirability trait linked to the FFQ to a greater extent than to the PFD [48-51]. There were some changes in the diet from shortly after surgery to 6 months (decreased intake of total and saturated fat and total n-6 PUFAs) and from six to 12 months (decreased intake of fish and fish products). The changes around 6 months postsurgery might be because the time-point coincided with the time of most extensive treatment for most of the patients. It has previously been demonstrated that breast cancer patients going through chemotherapy have an overall lower energy intake compared to healthy women, characterized by lower intake of protein, fat and alcohol $[52,53]$. On the contrary, chemotherapy has also been associated with an increased overall appetite and appetite for spicy and salty food [54]. Studies have further indicated that breast cancer patients are at risk for weight gain during and after chemotherapy, where both behavioural and physiological factors have been described to contribute to this weight gain $[54,55]$. Approximately 30 to $60 \%$ of breast cancer patients have previously reported that they changed their eating habits after breast cancer diagnosis [10]. 
However, most studies are based upon data gathered a long time after diagnosis and by questionnaires asking about changes in the diet or by semi-quantitative FFQs recalling diet both before and after diagnosis [10].

The sum n-3 PUFAs, EPA and DHA in serum phospholipids did not change from prediagnosis to 12 months postdiagnosis and Lindberg et al. have previously demonstrated that the long-term tracking (over 3 years) of the biomarker (plasma phospholipid n-3 PUFAs) was highly significant [22]. However, there are several factors that can potentially influence the level of serum phospholipid fatty acids besides the dietary intake [56]. The presence of a breast tumour may possibly affect the fatty acid composition in serum phospholipids because lipid metabolism may be altered in breast cancer [57-59], but it is uncertain if and how it may influence our results. Also, both chemo- and hormonal therapy have been demonstrated to affect lipid metabolism $[60,61]$. In this study, we stratified the patients according to treatment regime, to see if the treatment regime influenced the correlations between EPA/DHA intake and serum phospholipid fatty acids at any time point. The only significant difference found was the correlation between intake (PFD) and serum phospholipid EPA in the non-chemotherapy group ( $\rho$ 0.73) versus chemotherapy group $(\rho-0.05)$ at 6 months. The patients receiving chemotherapy had a significantly lower level of EPA in serum phospholipids, but there was no difference in the dietary intake. This single finding supports previous findings on chemotherapy and altered lipid metabolism $[60,61]$ and may indicate a limitation with our study. We also stratified the patients according to menopausal status, alcohol intake and BMI as both estrogen [16, 62], age $[62,63]$ and alcohol intake [64] have been suggested to influence serum phospholipid EPA/DHA, and obesity has been associated with altered lipid metabolism $[59,61]$. However, we found no significant differences between the strata. Importantly, the number of patients in this study was low, and other possible findings might be underestimated due to lack of power. Further, there are not made any adjustments for the multiple testing performed in this study.

In the present study the PFD, FFQ and biomarker were compared to each other at each time-point separately to demonstrate the differences seen throughout the year after diagnosis. The validation approach included comparisons of the dietary assessment methods medians, the correlations between the methods as well as with the biomarker and cross-classification of consumption/concentrations into tertiles [65]. Because three methods were compared in this study, the method of triads could have been performed. However, due to small sample size and low correlation coefficients at some time-points, it was concluded that it would probably have generated several Heywood cases (the appearance of validity coefficients greater than one) and would not have given any additional information. The method of triads was therefore not conducted [66].

\section{Conclusion}

The diet of patients with breast cancer or DCIS-grade III undergoing adjuvant treatment was quite stable and the intake of n-3 PUFAs did not change the year after surgery. However, our study suggests that the timing of mapping the diet is of importance. The FFQ and PFD in this study can be used to assess dietary intake of $n-3$ PUFAs and food sources of n-3 PUFAs in breast cancer patients during treatment, though the PFD shortly after surgery should be used with caution. This study demonstrates the importance of validating the dietary assessment tool within the patient-group it is intended to be used in as the patients' situation and diagnosis may influence the choice of tool and usage.

\section{Additional file}

Additional file 1: Table S1. The biomarker serum phospholipid fatty acids in $w t \%$ and quartiles $\left(P_{25}, 75\right), n=49$. Table S2. Spearman correlation coefficients $(\rho)$ for selected fatty acids from supplements, $n=49$. (DOCX 55 kb)

\section{Abbreviations \\ ALA: Alpha-linolenic acid; BMI: Body mass index; Cl: Confidence interval; DCIS: Ductal carcinoma in situ; DHA: Docosahexaenoic acid; DPA: Docosapentaenoic acid; E\%: Energy percent; EPA: Eicosapentaenoic acid; FFQ: Food frequency questionnaire; KBS: Kostberegningsystem; MUFA: Monounsaturated fatty acid; OT: Opposite tertile; $P_{25}$ : Percentile 25; $P_{75}$ : Percentile 75; PFD: Pre-coded food diary; PUFA: Polyunsaturated fatty acid; SD: Standard deviation; SFA: Saturated fatty acid; ST: Same tertile; WT\%: Weight percent}

\section{Acknowledgements}

We acknowledge each woman who participated in this clinical study. Further, we acknowledge our nurses Ragnhild Tveit and Alexandra Østgaard.

\section{Funding}

This work was funded by the Institute of Basic Medical Sciences, University of Oslo with supplementary funds from the Throne Holst Nutrition Research Foundation. Also, this study was supported by grants from South-East Norwegian Health Authority (grant 2012064), Norwegian Research Council (grant 213997) and Active Against Cancer Gjensidige Stiftelsen (grant 2012). The funders had no role in the design, collection of data, analysis, interpretation of data or writing of this article.

\section{Availability of data and materials}

The datasets used and analysed during the current study are not publicly available due to containing information that could compromise research patient privacy/consent but are available from the corresponding author on reasonable request.

\section{Authors' contributions}

The authors' roles in the study were as follows: SHB, IT and AH formulated the research questions, IT and $A H$ designed the study and SHB, IT, HF, VGF, GFB, ES and AH carried out the study. SHB and KSB analysed the data, SHB drafted the manuscript and all the authors critically revised and approved the final manuscript. 


\section{Ethics approval and consent to participate}

This study was conducted according to the guidelines laid down in the Declaration of Helsinki, and all procedures involving patients were approved by the Regional Committee for Medical Research Ethics (REK 2011/500). Written informed consent was obtained from all patients.

\section{Consent for publication}

Not applicable.

\section{Competing interests}

The authors declare that they have no competing interests.

\section{Publisher's Note}

Springer Nature remains neutral with regard to jurisdictional claims in published maps and institutional affiliations.

\begin{abstract}
Author details
${ }^{1}$ Department of Nutrition, Institute of Basic Medical Sciences, University of Oslo, PO Box 1046 Blindern, 0317 Oslo, Norway. ${ }^{2}$ The Cancer Centre, Oslo University Hospital Ullevål, Oslo, Norway. ${ }^{3}$ Department of Community Medicine, Faculty of Health Sciences, the Arctic University of Norway, Tromsø, Norway. ${ }^{4}$ Department of Physical Medicine and Rehabilitation, St. Olav University Hospital, Trondheim, Norway. ${ }^{5}$ Department of Neuromedicine and Movement Science, Norwegian University of Science and Technology, Trondheim, Norway. ${ }^{6}$ Department of Breast and Endocrine Surgery, Oslo University Hospital, Oslo, Norway. 'Department of Laboratory Medicine, St. Olav University Hospital, Trondheim, Norway. ${ }^{8}$ Department of Clinical and Molecular Medicine, Norwegian University of Science and Technology, Trondheim, Norway.
\end{abstract}

Received: 24 May 2018 Accepted: 1 October 2018

Published online: 17 October 2018

\section{References}

1. Fats and fatty acids in human nutrition. Report of an expert consultation. FAO Food Nutr Pap. 2010;91:1-166.

2. Calviello G, Serini S. Dietary Omega-3 polyunsaturated fatty acids and Cancer. In: Albini A, editor. Diet and Cancer 1. Netherlands: Springer; 2010.

3. Blucher C, Stadler SC. Obesity and breast cancer: current insights on the role of fatty acids and lipid metabolism in promoting breast cancer growth and progression. Front Endocrinol (Lausanne). 2017;8:293.

4. Monk JM, Turk HF, Liddle DM, De Boer AA, Power KA, Ma DW, Robinson LE. $\mathrm{N}-3$ polyunsaturated fatty acids and mechanisms to mitigate inflammatory paracrine signaling in obesity-associated breast cancer. Nutrients. 2014;6: 4760-93.

5. Al-Jawadi A, Moussa H, Ramalingam L, Dharmawardhane S, Gollahon L, Gunaratne P, Layeequr Rahman R, Moustaid-Moussa N. Protective properties of $\mathrm{n}-3$ fatty acids and implications in obesity-associated breast cancer. J Nutr Biochem. 2018:53:1-8.

6. Khankari NK, Bradshaw PT, Steck SE, He K, Olshan AF, Shen J, Ahn J, Chen Y, Ahsan $\mathrm{H}$, Terry MB, et al. Dietary intake of fish, polyunsaturated fatty acids, and survival after breast cancer: a population-based follow-up study on Long Island, New York. Cancer. 2015;121:2244-52.

7. Fabian CJ, Kimler BF, Hursting SD. Omega-3 fatty acids for breast cancer prevention and survivorship. Breast Cancer Res. 2015;17:62.

8. Makarem N, Chandran U, Bandera EV, Parekh N. Dietary fat in breast cancer survival. Annu Rev Nutr. 2013;33:319-48.

9. Liu J, Ma DW. The role of $\mathrm{n}-3$ polyunsaturated fatty acids in the prevention and treatment of breast cancer. Nutrients. 2014;6:5184-223.

10. Ghelfi F, Tieri M, Gori S, Nicolis F, Petrella MC, Filiberti A, Apolone G, Titta L. Do cancer patients change their diet in the e-health information era? A review of the literature and a survey as a proposal for the Italian population. Food Res Int. 2018;104:59-68.

11. Illner AK, Nothlings $U$, Wagner $K$, Ward $H$, Boeing $H$. The assessment of individual usual food intake in large-scale prospective studies. Ann Nutr Metab. 2010;56:99-105.

12. Nelson M, Black AE, Morris JA, Cole TJ. Between- and within-subject variation in nutrient intake from infancy to old age: estimating the number of days required to rank dietary intakes with desired precision. Am J Clin Nutr. 1989;50:155-67.
13. Tucker KL. Assessment of usual dietary intake in population studies of genediet interaction. Nutr Metab Cardiovasc Dis. 2007;17:74-81.

14. Riboli E, Ronnholm H, Saracci R. Biological markers of diet. Cancer Surv. 1987;6:685-718

15. Fekete K, Marosvolgyi T, Jakobik V, Decsi T. Methods of assessment of n-3 long-chain polyunsaturated fatty acid status in humans: a systematic review. Am J Clin Nutr. 2009;89:2070s-84s.

16. Hodson L, Skeaff CM, Fielding BA. Fatty acid composition of adipose tissue and blood in humans and its use as a biomarker of dietary intake. Prog Lipid Res. 2008;47:348-80.

17. Arab L. Biomarkers of fat and fatty acid intake. J Nutr. 2003;133(Suppl 3): 925s-32s.

18. Baylin A, Campos H. The use of fatty acid biomarkers to reflect dietary intake. Curr Opin Lipidol. 2006;17:22-7.

19. Serra-Majem L, Nissensohn M, Overby NC, Fekete K. Dietary methods and biomarkers of omega 3 fatty acids: a systematic review. Br J Nutr. 2012; 107(Suppl 2):S64-76.

20. Overby NC, Serra-Majem L, Andersen LF. Dietary assessment methods on $n$ 3 fatty acid intake: a systematic review. Br J Nutr. 2009;102(Suppl 1):S56-63.

21. Gibson RS. Principles of nutritional assessment. 2nd ed. Oxford: Oxford University Press; 2005.

22. Lindberg M, Midthjell K, Bjerve KS. Long-term tracking of plasma phospholipid fatty acid concentrations and their correlation with the dietary intake of marine foods in newly diagnosed diabetic patients: results from a follow-up of the HUNT study, Norway. Br J Nutr. 2013;109:1123-34.

23. Bjerve KS, Daae LN, Bremer J. The selective loss of lysophospholipids in some commonly used lipid-extraction procedures. Anal Biochem. 1974;58:238-45.

24. Andersen LF, Solvoll K, Johansson LR, Salminen I, Aro A, Drevon CA. Evaluation of a food frequency questionnaire with weighed records, fatty acids, and alpha-tocopherol in adipose tissue and serum. Am J Epidemiol. 1999;150:75-87.

25. Andersen LF, Tomten H, Haggarty P, Lovo A, Hustvedt BE. Validation of energy intake estimated from a food frequency questionnaire: a doubly labelled water study. Eur J Clin Nutr. 2003;57:279-84.

26. Nes M, Frost Andersen L, Solvoll K, Sandstad B, Hustvedt BE, Lovo A, Drevon CA. Accuracy of a quantitative food frequency questionnaire applied in elderly Norwegian women. Eur J Clin Nutr. 1992:46:809-21.

27. Andersen LF, Solvoll K, Drevon CA. Very-long-chain $n-3$ fatty acids as biomarkers for intake of fish and n-3 fatty acid concentrates. Am J Clin Nutr. 1996;64:305-11.

28. Andersen LF, Pollestad ML, Jacobs DR Jr, Lovo A, Hustvedt BE. Validation of a pre-coded food diary used among 13-year-olds: comparison of energy intake with energy expenditure. Public Health Nutr. 2005;8:1315-21.

29. Lillegaard IT, Andersen LF. Validation of a pre-coded food diary with energy expenditure, comparison of under-reporters $\mathrm{v}$. acceptable reporters. Br J Nutr. 2005;94:998-1003.

30. Myhre JB, Johansen AMW, Hjartaker A, Andersen LF. Relative validation of a pre-coded food diary in a group of Norwegian adults - comparison of underreporters and acceptable reporters. PLoS One. 2018;13:0202907.

31. Lillegaard IT, Overby NC, Andersen LF. Can children and adolescents use photographs of food to estimate portion sizes? Eur J Clin Nutr. 2005;59:611-7.

32. Tables of Sample Size Requirement for Estimating the Correlation Coefficient. https://www.statstodo.com/SSizCorr_Exp.php Accessed 23 May 2018.

33. da Silva DCG, Segheto W, de Lima MFC, Pessoa MC, Peluzio MCG, Marchioni DML, Cunha DB, Longo GZ: Using the method of triads in the validation of a food frequency questionnaire to assess the consumption of fatty acids in adults. J Hum Nutr Diet 2017;Published online 27. juli 2017. doi: 2010.1111/ jhn. 12497.

34. Hollis JL, Craig LC, Whybrow S, Clark H, Kyle JA, McNeill G. Assessing the relative validity of the Scottish collaborative group FFQ for measuring dietary intake in adults. Public Health Nutr. 2017;20:449-55.

35. Larkin FA, Metzner HL, Thompson FE, Flegal KM, Guire KE. Comparison of estimated nutrient intakes by food frequency and dietary records in adults. J Am Diet Assoc. 1989;89:215-23.

36. Praagman J, Adolphs AP, van Rossum CT, Sluijs I, van der Schouw YT, Beulens JW. Reproducibility and relative validity of a FFQ to estimate the intake of fatty acids. Br J Nutr. 2016;115:2154-61.

37. Segovia-Siapco G, Singh P, Jaceldo-Siegl K, Sabate J. Validation of a foodfrequency questionnaire for measurement of nutrient intake in a dietary intervention study. Public Health Nutr. 2007;10:177-84. 
38. Bingham SA, Gill C, Welch A, Day K, Cassidy A, Khaw KT, Sneyd MJ, Key TJ, Roe L, Day NE. Comparison of dietary assessment methods in nutritional epidemiology: weighed records $\mathrm{v}$. $24 \mathrm{~h}$ recalls, food-frequency questionnaires and estimated-diet records. Br J Nutr. 1994;72:619-43.

39. The Norwegian Directorate of Health Norkost 3 - En landsomfattende kostholdsundersøkelse blant menn og kvinner i Norge i alderen 18-70 år, 2010-11 (Norkost 3 - A Nationwide Dietary Survey Among 18-70 Year Old Men and Women in Norway, 2010-11).https://helsedirektoratet.no/Lists/ Publikasjoner/Attachments/301/Norkost-3-en-landsomfattendekostholdsundersokelse-blant-menn-og-kvinner-i-norge-i-alderen-18-70-ar2010-11-IS-2000.pdf Directorate of Health; 2012. Accessed 23.May 2018.

40. Hidaka BH, Li S, Harvey KE, Carlson SE, Sullivan DK, Kimler BF, Zalles CM, Fabian CJ. Omega-3 and omega- 6 fatty acids in blood and breast tissue of high-risk women and association with atypical cytomorphology. Cancer Prev Res (Phila). 2015;8:359-64.

41. Chajes V, Hulten K, Van Kappel AL, Winkvist A, Kaaks R, Hallmans G, Lenner P, Riboli E. Fatty-acid composition in serum phospholipids and risk of breast cancer: an incident case-control study in Sweden. Int J Cancer. 1999;83:585-90.

42. Wennberg M, Vessby B, Johansson I. Evaluation of relative intake of fatty acids according to the northern Sweden FFQ with fatty acid levels in erythrocyte membranes as biomarkers. Public Health Nutr. 2009;12:1477-84.

43. Kobayashi M, Jwa SC, Ogawa K, Morisaki N, Fujiwara T. Validity of a food frequency questionnaire to estimate long-chain polyunsaturated fatty acid intake among Japanese women in early and late pregnancy. J Epidemiol. 2017:27:30-5.

44. Zhang B, Wang P, Chen CG, He QQ, Zhuo SY, Chen YM, Su YX. Validation of an FFQ to estimate the intake of fatty acids using erythrocyte membrane fatty acids and multiple 3d dietary records. Public Health Nutr. 2010;13: 1546-52.

45. Arnaboldi P, Riva S, Crico C, Pravettoni G. A systematic literature review exploring the prevalence of post-traumatic stress disorder and the role played by stress and traumatic stress in breast cancer diagnosis and trajectory. Breast Cancer (Dove Med Press). 2017;9:473-85.

46. Maunsell E, Drolet M, Brisson J, Robert J, Deschenes L. Dietary change after breast cancer: extent, predictors, and relation with psychological distress. J Clin Oncol. 2002;20:1017-25.

47. Cade JE, Burley VJ, Warm DL, Thompson RL, Margetts BM. Food-frequency questionnaires: a review of their design, validation and utilisation. Nutr Res Rev. 2004;17:5-22

48. Di Noia J, Cullen KW, Monica D. Social desirability trait is associated with self-reported vegetable intake among women enrolled in the special supplemental nutrition program for women, infants, and children. J Acad Nutr Diet. 2016;116:1942-50.

49. Hebert JR. Social desirability trait: Biaser or driver of self-reported dietary intake? J Acad Nutr Diet. 2016;116:1895-8.

50. Hebert JR, Ebbeling CB, Matthews CE, Hurley TG, Ma Y, Druker S, Clemow L. Systematic errors in middle-aged women's estimates of energy intake: comparing three self-report measures to total energy expenditure from doubly labeled water. Ann Epidemiol. 2002;12:577-86.

51. Hebert JR, Clemow L, Pbert L, Ockene IS, Ockene JK. Social desirability bias in dietary self-report may compromise the validity of dietary intake measures. Int J Epidemiol. 1995;24:389-98.

52. de Vries YC, van den Berg MM, de Vries JH, Boesveldt $\mathrm{S}$, de Kruif JT, Buist $\mathrm{N}$, Haringhuizen A, Los M, Sommeijer DW, Timmer-Bonte JH, et al. Differences in dietary intake during chemotherapy in breast cancer patients compared to women without cancer. Support Care Cancer. 2017. https://doi.org/10. 1007/s00520-017-3668-x

53. Boltong A, Aranda S, Keast R, Wynne R, Francis PA, Chirgwin J, Gough K. A prospective cohort study of the effects of adjuvant breast cancer chemotherapy on taste function, food liking, appetite and associated nutritional outcomes. PLoS One. 2014;9:e103512.

54. Marinho EDC, Custodio IDD, Ferreira IB, Crispim CA, Paiva CE, Maia YCP. Impact of chemotherapy on perceptions related to food intake in women with breast cancer: a prospective study. PLoS One. 2017;12:e0187573.

55. Thomson ZO, Reeves MM. Can weight gain be prevented in women receiving treatment for breast cancer? A systematic review of intervention studies. Obes Rev. 2017;18:1364-73.

56. Simopoulos AP. Essential fatty acids in health and chronic disease. Am J Clin Nutr. 1999;70:560S-9S.

57. Baumann J, Sevinsky C, Conklin DS. Lipid biology of breast cancer. Biochim Biophys Acta. 2013;1831:1509-17.
58. Saadatian-Elahi M, Norat T, Goudable J, Riboli E. Biomarkers of dietary fatty acid intake and the risk of breast cancer: a meta-analysis. Int J Cancer. 2004; 111:584-91.

59. Long J, Zhang CJ, Zhu N, Du K, Yin YF, Tan X, Liao DF, Qin L. Lipid metabolism and carcinogenesis, cancer development. Am J Cancer Res. 2018:8:778-91.

60. Felicetti F, Fortunati N, Brignardello E. Cancer survivors: an expanding population with an increased cardiometabolic risk. Diabetes Res Clin Pract. 2018;143:432-42.

61. Dieli-Conwright CM, Wong L, Waliany S, Bernstein L, Salehian B, Mortimer JE. An observational study to examine changes in metabolic syndrome components in patients with breast cancer receiving neoadjuvant or adjuvant chemotherapy. Cancer. 2016;122:2646-53.

62. Kitson AP, Stroud CK, Stark KD. Elevated production of docosahexaenoic acid in females: potential molecular mechanisms. Lipids. 2010;45:209-24.

63. Otsuka R, Kato $Y$, Imai T, Ando F, Shimokata H. Higher serum EPA or DHA, and lower ARA compositions with age independent fatty acid intake in Japanese aged 40 to 79. Lipids. 2013;48:719-27.

64. di Giuseppe R, de Lorgeril M, Salen P, Laporte F, Di Castelnuovo A, Krogh V, Siani A, Arnout J, Cappuccio FP, van Dongen M, et al. Alcohol consumption and $n-3$ polyunsaturated fatty acids in healthy men and women from 3 European populations. Am J Clin Nutr. 2009;89:354-62.

65. Serra-Majem L, Frost Andersen L, Henrique-Sanchez P, Doreste-Alonso J, Sanchez-Villegas A, Ortiz-Andrelluchi A, Negri E, La Vecchia C. Evaluating the quality of dietary intake validation studies. Br J Nutr. 2009;102(Suppl 1):S3-9.

66. Ocke MC, Kaaks RJ. Biochemical markers as additional measurements in dietary validity studies: application of the method of triads with examples from the European prospective investigation into cancer and nutrition. Am J Clin Nutr. 1997;65:1240s-5s.

\section{Ready to submit your research? Choose BMC and benefit from:}

- fast, convenient online submission

- thorough peer review by experienced researchers in your field

- rapid publication on acceptance

- support for research data, including large and complex data types

- gold Open Access which fosters wider collaboration and increased citations

- maximum visibility for your research: over $100 \mathrm{M}$ website views per year

At $\mathrm{BMC}$, research is always in progress.

Learn more biomedcentral.com/submissions 